\title{
VIRTIS on Venus Express: retrieval of real surface emissivity on global scales
}

\author{
Gabriele E. Arnold ${ }^{\mathrm{a}}$, David Kappel ${ }^{\mathrm{a}}$, Rainer Haus ${ }^{\mathrm{b}}$, Laura Telléz Pedroza ${ }^{\mathrm{a}, \mathrm{c}}$, Giuseppe \\ Piccioni $^{\mathrm{d}}$, and Pierre Drossart ${ }^{\mathrm{e}}$ \\ ${ }^{a}$ Deutsches Zentrum für Luft- und Raumfahrt e.V. (DLR), Institute of Planetary Research, \\ Rutherfordstrasse 2, 12489 Berlin, Germany; \\ ${ }^{\mathrm{b}}$ Westfälische Wilhelms-Universität, Institute of Planetology, Wilhelm-Klemm-Str. 10, 48149 \\ Münster, Germany; \\ ${ }^{c}$ University Potsdam, Institute of Earth and Environmental Science, Karl-Liebknecht-Str. 24-25, \\ 14476 Potsdam, Germany; \\ ${ }^{\mathrm{d}}$ Istituto di Astrofisica e Planetologia Spaziali (IAPS), INAF, Via Fosso del Cavaliere 100, 00133, \\ Roma, Italy; \\ 'Laboratoire d'Études Spatiales et d'Instrumentation en Astrophysique (LESIA), Observatoire de \\ Paris, 5 Place Jules Janssen, 92195, Meudon, France. \\ *Gabriele.Arnold@dlr.de; phone +49-3067055370; fax + 49-3067055303
}

\begin{abstract}
The extraction of surface emissivity data provides the data base for surface composition analyses and enables to evaluate Venus' geology. The Visible and InfraRed Thermal Imaging Spectrometer (VIRTIS) aboard ESA's Venus Express mission measured, inter alia, the nightside thermal emission of Venus in the near infrared atmospheric windows between 1.0 and $1.2 \mu \mathrm{m}$. These data can be used to determine information about surface properties on global scales. This requires a sophisticated approach to understand and consider the effects and interferences of different atmospheric and surface parameters influencing the retrieved values. In the present work, results of a new technique for retrieval of the $1.0-1.2$ $\mu \mathrm{m}-$ surface emissivity are summarized. It includes a Multi-Window Retrieval Technique, a Multi-Spectrum Retrieval technique (MSR), and a detailed reliability analysis. The MWT bases on a detailed radiative transfer model making simultaneous use of information from different atmospheric windows of an individual spectrum. MSR regularizes the retrieval by incorporating available a priori mean values, standard deviations as well as spatial-temporal correlations of parameters to be retrieved. The capability of this method is shown for a selected surface target area. Implications for geologic investigations are discussed. Based on these results, the work draws conclusions for future Venus surface composition analyses on global scales using spectral remote sensing techniques. In that context, requirements for observational scenarios and instrumental performances are investigated, and recommendations are derived to optimize spectral measurements for Venus' surface studies.
\end{abstract}

Keywords: Planetary remote sensing, terrestrial planets, Venus, spectroscopy.

\section{INTRODUCTION}

Venus is called a "sister planet" of Earth, and thus it has been an important object of planetary research and comparative planetology. Although Venus and Earth formed close together 4.6 billion years ago, they followed dramatically different evolutionary paths ${ }^{1}$. The current hostile environmental conditions on Venus are dominated by a massive atmosphere, consisting mainly of $\mathrm{CO}_{2}$ and increasing the surface temperature and pressure up to $735 \mathrm{~K}$ and 92 bar respectively ${ }^{2,3}$. These conditions are known to be driven by a runaway greenhouse effect and an atmospheric superrotation ${ }^{4}$. Therefore,

Infrared Remote Sensing and Instrumentation XXIII, edited by Marija Strojnik Scholl,

Gonzalo Páez, Proc. of SPIE Vol. 9608, 960806 - @ 2015 SPIE

CCC code: $0277-786 X / 15 / \$ 18 \cdot$ doi: $10.1117 / 12.2186619$

Proc. of SPIE Vol. 9608 960806-1 
Venus' atmosphere went along a path towards its present environment different from Earth. To understand the underlying evolutionary processes requires deeper insights into the structure of the planetary body. One promising approach is the study of the geologic, morphologic, stratigraphic, and compositional structure of the Venusian surface. The thick and opaque atmosphere of Venus prevents dayside surface observations at visible wavelengths. Most of the current knowledge about Venus' surface results from radar ${ }^{5}$ and a few lander ${ }^{6}$ measurements. The discovery of nightside nearinfrared atmospheric emission windows between 0.8 and $2.5 \mu \mathrm{m}$ provided a new technique for studying the lower atmosphere of Venus down to the surface ${ }^{7}$. Flybys of Galileo/NIMS and Cassini/VIMS ${ }^{8,9}$ made use of these windows adding new data of Venus' lower atmosphere and surface. The Visual and InfraRed Thermal Imaging Spectrometer (VIRTIS) aboard the ESA mission Venus Express was the first spectrometer continuously measuring the nightside thermal emission orbiting the planet from 2006 until $2014^{10}$. The VIRTIS data have been used developing a technique to retrieve Venus' surface emissivity. Knowledge of surface emissivity enables to analyze surface properties like temperatures, potential surface materials, and surface roughness ${ }^{11,12}$. This paper summarizes the approach and exemplary results for the Themis Regio region on Venus.

\section{VIRTIS ON VENUS EXPRESS}

The Visible and InfraRed Thermal Imaging Spectrometer (VIRTIS) on ESA's Venus Express (VEX) provided an enormous amount of data including a three-dimensional view of the atmosphere and information on global surface properties of the planet. VIRTIS/VEX is an imaging spectrometer combining three data channels in one compact instrument ${ }^{13,14,15} 16$. Two of them are devoted to spectral mapping at moderate spectral resolution (Mapper optical subsystem "-M") in the range from 0.27 to $5.10 \mu \mathrm{m}$. A spectrally resolved VIRTIS-M image is called "data cube". The third channel is devoted to spectroscopy (High resolution optical subsystem "-H") in the spectral range from 2 to $5 \mu \mathrm{m}$. The $-\mathrm{H}$ field of view is approximately centered in the middle of the $-\mathrm{M}$ image providing high spectral resolution spectra in this small portion of image ${ }^{13}$.

VIRTIS consists of four modules: the Optics Modules -M and -H, the two Proximity Electronics Modules (PEM), and the Main Electronics (ME). The Optics Modules are externally mounted on the $-X$ panel of the spacecraft with the Optical Heads co-aligned in $+Z$ direction (planet view). VIRTIS-M is characterized by a single optical head consisting of a Shafer telescope combined with an Offner imaging spectrometer and by two focal plane arrays (FPA). VIRTIS-H is a high spectral resolution Echelle cross-dispersed spectrometer using prism and grating for dispersion ${ }^{13,14,15,16}$.

The spectral sampling of $-\mathrm{M}$ is about $2 \mathrm{~nm}$ below $1 \mu \mathrm{m}$ and $10 \mathrm{~nm}$ from 1 to $5.10 \mu \mathrm{m}$, while for $-\mathrm{H}$ it is about $1.6 \mathrm{~nm}$, depending on wavelength and scattering order. VIRTIS-M-VIS uses a Si CCD (Thomson TH7896) for the range between 0.28- $1.1 \mu \mathrm{m}$. The IR FPAs of $-\mathrm{M}$ and $-\mathrm{H}$ are housed on bi-dimensional $\mathrm{HgCdTe}$ arrays designed to provide high sensitivity and low dark current ( $1 \mathrm{fA}$ at $80 \mathrm{~K})$. They are cooled to $85 \mathrm{~K}$ by an active cooler. VIRTIS-M and $-\mathrm{H}$ spectrometers themselves are cooled down to $135 \mathrm{~K}$ by means of a radiator reducing the background level of thermal radiation $^{13,14,15,16}$.

\section{RETRIEVAL METHOD}

Venus surface emissivity retrieval bases on a detailed radiative transfer simulation model ("forward model") ${ }^{12,17,18,19}$, a multi-spectrum retrieval technique (MSR) ${ }^{18,19,20}$, and a detailed error analysis ${ }^{19,20}$. The radiative transfer model simulates the radiance spectra. It considers absorption, emission, and multiple scattering by gaseous and particulate constituents of the atmosphere. MSR regularizes the retrieval by incorporation of available a priori mean values and standard deviations of parameters to be retrieved and physically reasonable spatial-temporal a priori correlations. Moreover, it can retrieve parameters that are common to a set of spectra. The retrieval pipeline results in emissivity maps relative to a reference value.

\subsection{Radiative transfer simulation model and single-spectrum retrieval}

Venus' hot surface emits IR radiation, depending on its temperature and emissivity. As it wells upward, this radiation gets mixed with thermal emissions of the atmosphere and is partially absorbed and multiply scattered by atmospheric gases 
and clouds. These processes also lead to a horizontal blurring of the information on the spatial origin of the surface properties imprinted in the IR radiance on a scale of $100 \mathrm{~km}^{21}$. Since surface signatures are far outweighed by scattered sunlight on the dayside, only nightside measurements can be used to derive surface data. In the VIRTIS-M-IR spectral range, nightside spectral windows at 1.02, 1.10, and $1.18 \mu \mathrm{m}$ (the "surface windows") sound the surface, while additional windows between 1.3 and $2.6 \mu \mathrm{m}$ (the "deep atmosphere windows") sound the deep atmosphere around $20-40 \mathrm{~km}$ altitude (see Fig. 1). An additional window at $1.28 \mu \mathrm{m}$ is not taken into account here, since it is contaminated by $\mathrm{O}_{2}$ nightglow. The measured radiance in the surface windows depends also on atmospheric properties that have to be derived from the deep atmosphere windows.

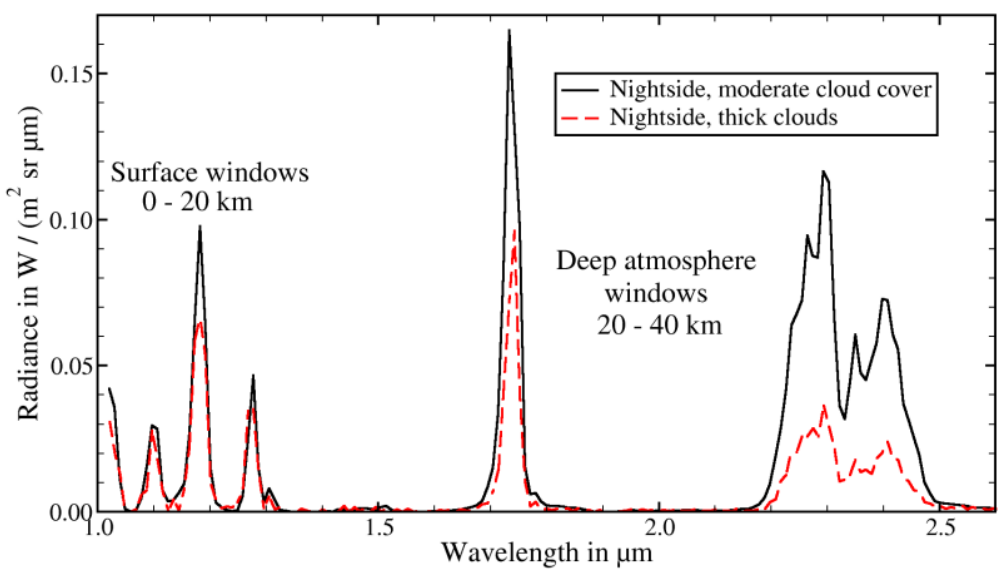

Figure 1. Calibrated and preprocessed ${ }^{22,23}$ VIRTIS-M-IR spectra of Venus' nightside (cubes VI0320_01 at $240.5^{\circ} \mathrm{E}, 31.1^{\circ} \mathrm{S}$, and VI0327_02 at $\left.240.5^{\circ} \mathrm{E}, 38.5^{\circ} \mathrm{S}\right)$.

To derive quantitative information from a measured VIRTIS-M-IR spectrum, it is necessary to compute synthetic spectra for given sets of atmospheric, surface, and instrumental parameters. The observation geometry as well as certain physical and optical properties of the atmospheric gases and clouds have to be known in addition. Haus and Arnold (2010) ${ }^{12}$ with updates ${ }^{17}$ described a radiance transfer simulation program, which takes the aforementioned radiative processes into account. It is an LTE line-by-line model based on the discrete ordinate method DISORT ${ }^{24}$. It pays particular attention to the extreme environmental conditions close to Venus' surface and is suitable for plane-parallel geometries. A further update $^{18}$ based on LIDORT ${ }^{25}$ enables the computation of analytic derivatives of the simulated radiance spectra with respect to many parameters to be retrieved.

Starting with an initial guess of the state vector, i.e. the vector formed by the wanted parameters, a retrieval algorithm compares the corresponding simulated spectrum to the measured spectrum and iteratively modifies the state vector until the simulated spectrum well fits the measured one. The state vector leading to the best fit can be regarded as an estimate of the actual states of the atmosphere and surface that led to the measured spectrum. But since different state vectors can describe the same measured spectrum equally well, the retrieval has to be regularized. A priori mean values and standard deviations of the retrieval parameters as well as information on the measurement and simulation errors of the spectra can be taken into account in the frame of a retrieval algorithm based on Bayesian regularization. The algorithm searches for the location of the maximum of the corresponding Bayesian a posteriori probability distribution function. Of all state vectors that well describe the measured spectrum; this location is the one that is the most compatible with the a priori and error information ${ }^{26}$. This way, unreasonable state vectors can be excluded from the outset.

VIRTIS-M-IR single-spectrum information content is quite limited, which is why there are always surface and atmospheric parameters that cannot be derived from the measured spectra with sufficient reliability. These parameters have to be set to reasonable values compatible with current knowledge on atmospheric and surface conditions and allow the computation of realistic synthetic spectra. When these assumed interfering parameters deviate from the true physical values, this may cause severe surface emissivity retrieval errors, even though the fits well match the measured spectra ${ }^{19}$. 


\subsection{Multi-spectrum retrieval technique}

The situation discussed in the previous section can be improved by including further a priori information. Due to the physical drive to balance thermodynamic disequilibria, spatially or temporally contiguous VIRTIS-M-IR measurements are unlikely to originate from completely unrelated single-spectrum state vectors. To describe such correlations, the recently developed multi-spectrum retrieval algorithm $\mathrm{MSR}^{18}$ can take a priori correlation lengths and times of the retrieval parameters into account. For this purpose, a set of many spectra is regarded as a single meta-measurement, defining a corresponding Bayesian a posteriori probability distribution function. MSR can also retrieve parameter vectors that are common to the utilized set of spectra. Incorporating this kind of a priori data, unreasonable spatial-temporal state vector distributions (like unphysical jumps between different subsidiary single-spectrum solutions) can be excluded from the outset, and the reliability of the retrieved parameters can be increased. In particular, an emissivity map of a given target on Venus' surface can be retrieved at each surface window with MSR as parameter vector that is common to many VIRTIS-M-IR cubes covering this target, neglecting geologic activity and thereby surface emissivity variations with time.

It turns out that even with MSR, absolute emissivity values are difficult to obtain due to strong interferences from other parameters. Moreover, retrieved emissivity maps exhibit trends with surface elevation and latitude. These trends were interpreted $^{20}$ to be due to imperfect radiative transfer simulations with respect to the extinction properties of the deep atmosphere and to deviations between time-averaged true and assumed interfering parameters. The latter deviations were argued to be spatially slowly varying and only in latitude direction, motivated by the symmetries and spatial scales of the global atmospheric circulation. After removal of the emissivity trends in a post-processing step, it was observed ${ }^{20}$ that the magnitude of the resulting spatial emissivity fluctuations around their mean value increased with increasing mean value. This is because the radiance response to small emissivity perturbations around an emissivity base value decreases for increasing emissivity base value. The true mean value of the map cannot be retrieved from the data with the described techniques. To still obtain well-defined quantitative emissivity data maps, the fluctuation behavior was quantified, and the de-trended retrieved emissivity maps were transformed to have an emissivity mean value that attains a fixed predefined reference emissivity of 0.5 . The results no longer represent absolute emissivity values but relative ones and were called "renormalized emissivity maps" or "relative emissivity maps". They can be referred to another reference emissivity if needed, for instance in the case when the absolute emissivity at a given point of the target should become known (e.g. in the form of ground truth data).

\section{SURFACE EMISSIVITY DATA}

For a surface target at Themis Regio, it was demonstrated ${ }^{20}$ with many tests that the renormalized emissivity maps at the three surface windows are reasonably independent of the selection of the utilized measured spectra as well as of modifications to various interfering atmospheric, surface, and instrumental parameters and to selected details of the retrieval pipeline and data calibration and preprocessing. A statistical evaluation of these tests according to mathematically founded error scaling and adding rules yielded double standard deviation error margins of about 3\%, $8 \%$, and $4 \%$ for the renormalized retrieved emissivities in the surface windows at $1.02,1.10$, and $1.18 \mu \mathrm{m}$, respectively, when each surface bin is covered by 64 measurements and the reference emissivity is set to 0.5 . The renormalized emissivities were interpreted as spatial variations relative to the reference emissivity. However, possible (but unlikely) real trends of the true emissivities with topography or latitude cannot be reflected by renormalized retrieved emissivities.

Spatial variations of the $1.02 \mu \mathrm{m}$ surface emissivity of $20 \%$ that correspond to the difference between unweathered granitic and basaltic rocks would be easily detectable, but such variations were ruled out for the studied target area at Themis Regio. Emissivity anomalies of up to $8 \%$ were detected at both 1.02 and $1.18 \mu \mathrm{m}$. No anomalies were identified at $1.10 \mu \mathrm{m}$, but anomalies exceeding the determined error level were excluded ${ }^{20}$. 


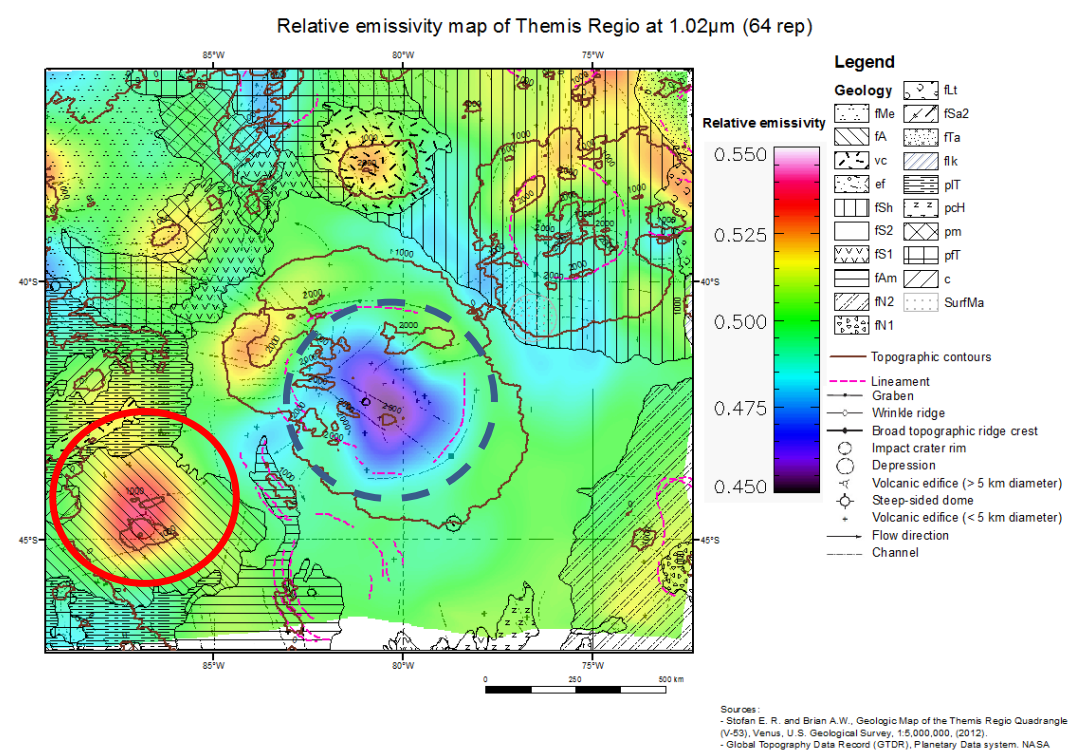

Figure 2. $1.02 \mu \mathrm{m}$ emissivity map ${ }^{20}$ overlaid onto the geologic map ${ }^{27}$ with topographic contoures ${ }^{28}$. Right: Legend, geology, and color bar for the retrieved emissivity. Red circle: Abeona Mons; blue circle (dashed): Shiwanokia Corona ${ }^{29}$.

Fig. 2 shows the emissivity map of the Themis Regio at $1.02 \mu \mathrm{m}$ relative to the reference emissivity 0.5 . It was determined as described above, where each surface spot was covered by 64 measurements. Themis Regio is a highland region classified as a corona-dominated hotspot rise ${ }^{30}$. The target area includes geologic structures like the Shiwanokia Corona with steep sided domes, Shulamite Coronae, Abeona and Mertseger Mons, impact craters like Kenny, Aksentyeva, and Sabin with a dark parabola, graben, and wrinkle ridges. The map displays clear spatial variations of surface emissivity at $1.02 \mu \mathrm{m}$. These "anomalies" can be caused by local changes in composition, age, and/or textural differences of the surface (smooth fresh vs. rough old and weathered areas $)^{20,29}$. Areas with low emissivity are associated with the central Shiwanokia Corona (see Fig. 2, blue circle), which is a complex and old geologic feature. In contrast, e.g. an increase of emissivity occurs along the flanks and hills of Abeona Mons (Fig. 2, red circle), which correlates rather with relatively young units interpreted to be of volcanic origin ${ }^{31}$.

\section{CONCLUSION}

The VIRTIS observations on ESA's Venus Express mission provided a new data set enabling to retrieve the relative surface emissivity in the 1 to $1.2 \mu \mathrm{m}$ region. A complex radiative transfer simulation model and a multi-spectrum retrieval technique were developed for this purpose. Additionally, a comprehensive error analysis was performed. This approach enabled to validly assess real global-scale surface near-infrared emissivity anomalies for the first time. Comparison of the retrieved emissivity data with surface features revealed by Magellan radar observations in the Themis Regio terrain on Venus allows the discussion of relevant geologic surface processes. Since the reliability of emissivity retrieval from nightside near-infrared spectral measurements depends on the repetition rate of observations covering the same surface footprint, future space borne spectroscopic experiments require a sophisticated operation strategy with a high coverage repetition rate and including also areas at high altitudes and the older tessera terrains. Furthermore, an increased sensitivity and improved spectral resolution within the $0.8-5.0 \mu \mathrm{m}$ range will help to improve the atmospheric model and the surface data extraction.

\section{ACKNOWLEDGEMENTS}

We acknowledge ASI, CNES to support the program and DLR and DFG for funding this work. We also acknowledge the work of the VIRTIS/VEX team and also the entire Venus Express team. 


\section{REFERENCES}

[1] Basilevsky, A. T. and Head J. W., “The geologic history of Venus: A stratigraphic view”, J. Geophys. Res. 103(E4), 8531-8544, doi:10.1029/98JE00487 (1998).

[2] Hunten, D.M., “Atmospheric evolution of terrestrial planets”, Science 259, 915-920 (1993).

[3] Kliore, A.J. et al., "The Venus International Reference Atmosphere", Adv. Space Res. 5(11), 1-305 (1985).

[4] Taylor, F.W., "Venus before Venus Express", Planet. Space Sci. 54, 1249-1262 (2006).

[5] Leberl, F.W. et al., "Surface topography reconstruction of Venus from the Magellan mission", Int. Archives of Photogrammetry and Remote Sensing XXXI, B4, 491-496 (1996).

[6] Basilevsky, A.T. et al., "The surface of Venus as revealed by the Venera Landings, Part II, Bull. Am. Geol. Soc. 96, 137-144 (1985).

[7] Allen, D.A., "The dark side of Venus", Icarus 69 (2), 221-229, doi:10.1016/0019-1035(87)90101-1 (1987).

[8] Carlson, R.W. et al., "Galileo infrared imaging spectroscopy measurements at Venus”, Science 253 (5027), 15411548 (1991).

[9] Baines, K.H. et al., "Detection of sub-micron radiation from the surface of Venus by Cassini/VIMS", Icarus 48(1), 307-311, doi:10.1006/icar.2000.6519 (2000).

[10] Titov, D. et al., "Introduction to the special section on Venus Express: results of the Nominal Mission", J. Geophys. Res. 113 (E00B19), 1-2 (2008).

[11] Arnold, G. et al., "Venus surface data extraction from VIRTIS/Venus Express measurements: Estimation of a quantitative approach", J. Geophys. Res. 13(E00B10), doi:10.1029/2008JE003087 (2008)

[12] Haus, R. and Arnold, G., "Radiative transfer in the atmosphere of Venus and application to surface emissivity retrieval from VIRTIS/VEX measurements", Planet. Space Sci. 58 (12), 1578-1598, doi:10.1016/j.pss2010.08.001 (2010).

[13] Drossart, P. et al., "VIRTIS imaging spectrometer for ESA/Venus Express mission", SPIE 5543, 175-185 (2004).

[14] Drossart, P. et al., "Scientific goals for the observation of Venus by VIRTIS on ESA/Venus Express mission", Planet. Space Sci. 55, 1653-1672, doi:10.1016/j.pss.2007.01.003 (2007).

[15] Piccioni, G. et al., "South-polar features on Venus similar to those near the north pole", Nature 450 (7170), 637-640, doi:10.1038/nature06209 (2007).

[16] Arnold, G.E., "Exploring the solar system: the view of planetary surfaces with VIS/IR remote sensing methods", Proc. SPIE 8154, 815402, 19 pages, doi:10.1117/12.897759 (2011).

[17] Haus, R. et al., "Self-consistent retrieval of temperature profiles and cloud structure in the northern hemisphere of Venus using VIRTIS/VEX and PMV/VENERA-15 radiation measurements", Planet. Space Sci. 89, 77-101, doi:10.1016/jpss.2013.09.020 (2013).

[18] Kappel, D., "MSR, a multi-spectrum retrieval technique for spatially-temporally correlated or common Venus surface and atmosphere parameters”, J. Quant. Spectrosc. Ra. 133, 153-176, doi:10.1016/j.jqsrt.2013.07.025 (2014).

[19] Kappel, D., et al., "Error analysis for retrieval of Venus' IR surface emissivity from VIRTIS/VEX measurements", Planet. Space Sci. 113-114, 49-65, doi:10.1016/j.pss.2015.01.014 (2015).

[20] Kappel, D. et al., "Multi-spectrum retrieval of Venus IR surface emissivity maps from VIRTIS/VEX nightside measurements at Themis Regio", sumitted to Icarus (2015).

[21] Moroz, V.I., "Estimates of visibility of the surface of Venus from decent probes and balloons", Planet. Space Sci. 50, 287-297, doi:10.1016/S0032-0633(01)00128-3 (2002).

[22] Cardesin-Moinelo, A. et al., "Calibration of Hyperspectral Imaging Data: VIRTIS-M Onboard Venus Express", IEEE Trans. Geosci. Remote 48, 3941-3950, doi:10.1109/TGRS.2010.2064325 (2010).

[23] Kappel, D. et al., "Refinements in the data analysis of VIRTIS-M-IR Venus nightside spectra", Adv. Space Res. 50 (2), 228-255, doi:10.1016/j.asr.2012.03.029 (2012).

[24] Stamnes, K. et al., "Numerically stable algorithm for discrete-ordinate-method radiative transfer in multiple scattering and emitting layered media", Appl. Opt. 27 (12), 2502-2509, doi:10.1364/AO.27.002502 (1988).

[25] Spurr, R., "LIDORT and VLIDORT: Linearized pseudo-spherical scalar and vector discrete ordinate radiative transfer models for use in remote sensing retrieval problems", In: Light Scattering Reviews 3, A. A. Kokhanovsky [Ed.], Springer, Berlin Heidelberg, 229-275, ISBN: 978-3-540-48305-2, doi:10.1007/978-3-540-48546-9_7 (2008).

[26] Rodgers, C. D., "Inverse Methods for Atmospheric Sounding: Theory and Practice (Series on Atmospheric, Oceanic and Planetary Physics)", World Scientific Publishing Company, Singapore, ISBN: 981022740X (2000).

[27] Stofan, E. R. and Brian, A. W., "Geologic map of the Themis Regio quadrangle (V-53), Venus", http://pubs.usgs.gov/sim/3165/, US Geological Survey Scientific Investigations Map 3165 (2012). 
[28] Ford, P. G. and Pettengill, G. H., "Venus Topography and Kilometer-Scale Slopes". J. Geophys. Res.: Planets 97 (E8), 13103-13114, doi:10.1029/92JE01085 (1992).

[29] Arnold, G. et al., "Retrieval and study of near-infrared emissivity maps of Themis Regio on Venus with VIRTIS-M (Venus Express", Proceedings of EPSC, EPSC2015-160 (2015).

[30] Stofan, E.R. et al., "Large topographic rises on Venus: implications for a mantle upwelling", J. Geophys. Res. 100, 23, 317- 23,325, doi:10.1029/95JE01834 (1995).

[31] Smrekar, S.E. et al., "Recent hot-spot volcanism on Venus from VIRTIS emissivity data", Science 328 (5978), 605 608, doi:10.1126/science.1186785 (2010). 